\title{
Weed Management in White Bean with Variable Doses of $S$-Metolachlor and Halosulfuron Applied Preemergence
}

\author{
Nader Soltani*, Christy Shropshire, Peter H. Sikkema \\ University of Guelph Ridgetown Campus, Ridgetown, Canada \\ Email: *soltanin@uoguelph.ca
}

How to cite this paper: Soltani, N., Shropshire, C. and Sikkema, P.H. (2019) Weed Management in White Bean with Variable Doses of $\mathcal{S}$-Metolachlor and Halosulfuron Applied Preemergence. Agricultural Sciences, 10, 1453-1464.

https://doi.org/10.4236/as.2019.1011106

Received: October 26, 2019

Accepted: November 17, 2019

Published: November 20, 2019

Copyright $\odot 2019$ by author(s) and Scientific Research Publishing Inc. This work is licensed under the Creative Commons Attribution International License (CC BY 4.0).

http://creativecommons.org/licenses/by/4.0/

\begin{abstract}
Five experiments were conducted in Ontario, Canada from 2016 to 2018 to determine how doses of $S$-metolachlor and halosulfuron applied preemergence (PRE) should be adjusted to control specific weed species in white bean. $S$-metolachlor, halosulfuron, and $S$-metolachlor + halosulfuron caused minimal ( $1 \%$ to $4 \%)$ injury in white bean. Weed interference reduced white bean yield $54 \%$. On average, weed interference with $S$-metolachlor and halosulfuron decreased yield $34 \%$ and $29 \%$, respectively. In contrast, white bean seed yield was similar to the weed-free control with the $S$-metolachlor + halosulfuron tankmixes. $S$-metolachlor applied alone controlled $A$. theophrasti, A. retroflexus, $A$. artemisiifolia, $C$. album, E. crus-galli and $S$. viridis $0 \%$ to $3 \%, 78 \%$ to $93 \%, 0 \%$ to $9 \%, 5 \%$ to $15 \%, 97 \%$ to $99 \%$ and $96 \%$ to $98 \%$, respectively. Halosulfuron applied alone controlled $A$. theophrasti, $A$. retroflexus, $A$. artemisiifolia, C. album, E. crus-galli and $S$. viridis $39 \%$ to $87 \%, 93 \%$ to $99 \%$, $64 \%$ to $88 \%, 34 \%$ to $59 \%, 10 \%$ to $30 \%$ and $13 \%$ to $35 \%$, respectively. $S$-metolachlor + halosulfuron tankmixes controlled $A$. theophrasti, A. retroflexus, $A$. artemisiifolia, C. album, E. crus-galli and S. viridis $47 \%$ to $94 \%, 98 \%$ to $100 \%$, $78 \%$ to $94 \%, 37 \%$ to $78 \%, 94 \%$ to $98 \%$ and $91 \%$ to $96 \%$, respectively. Weed density and biomass reductions with the herbicides evaluated followed the same pattern as visible weed control assessments. Results from this study indicate that doses of $S$-metolachlor and halosulfuron, when applied as a tankmix, should be adjusted based on a weed species composition in each individual white bean field.
\end{abstract}

\section{Keywords}

Biomass, Density, Dry Bean, Maturity, Seed Yield, Tolerance, Weed Control 


\section{Introduction}

Dry bean (Phaseolus vulgaris L.) is popular legume crop grown in Ontario. Approximately $80 \%-90 \%$ of dry bean harvested in Ontario is exported out of the province [1]. White bean has been produced in the province since the early 1900 's and over the years has become the most popular dry bean market class grown [1]. In 2018, approximately 63,000 tonnes of white beans were produced from 22,000 ha in Ontario with a value of nearly $\$ 49$ million [2]. Controlling weeds is one of the most important concerns for white bean production in Ontario.

Typical problem weeds for white bean producers in Ontario include Abutilon theophrasti Medic. (velvetleaf), Amaranthus retroflexus L. (redroot pigweed), Ambrosia artemisiifolia L. (common ragweed), Chenopodium album L. (common lambsquarters), Sinapis arvensis L. (wild mustard), Polygonum persicaria L. (ladysthumb), Eastern black nightshade (Solanum ptycanthum Dun.), Xanthium strumarium L. (cocklebur), Digitaria sanguinalis (L.) Scop. (large crabgrass), Setaria viridis (L.) Beauv. (green foxtail), and Echinochloa crus-galli (L.) P. Beauv. (barnyardgrass) [3]. These problematic weeds generally germinate early in the season and are fast growing thereby outcompeting the slower growing white bean plants for irradiance, moisture and nutrients resulting in substantial yield losses [4]. White bean seed yield losses have been reported to be $68 \%$ to $81 \%$ in white bean from weed interference [5]-[12]. There are currently few herbicide choices that producers can choose from to control these problematic weed species in white bean.

Halosulfuron is a recently registered sulfonyl-urea herbicide for broadleaved weed control in white bean in Ontario (OMAFRA 2018). Major weeds controlled with halosulfuron includes $A$. theophrasti, $C$. album, $S$. arvensis, P. persicaria, $A$. retroflexus and $X$. strumarium, including biotypes that are resistant to Group 5 (triazine) herbicides [13] [14]. There is little activity with halosulfuron against grass weed species at doses registered in white bean (OMAFRA 2018). Therefore, halosulfuron needs to be used along with a graminicide to provide broadspectrum control of problematic weeds in white bean [3].

$S$-metolachlor (the active of isomer of metolachlor) is a chloroacetanilide herbicide that is registered in white bean to control of key weeds in Ontario including Echinochloa spp., Setaria spp., Panicum spp., Digitaria spp., Solanum spp. and Amaranthus spp. [15]. $S$-metolachlor tank mixed with halosulfuron can control troublesome grass and broadleaved weeds (including Group 5 resistant biotypes) in white bean.

The $S$-metolachlor label has a dose range of 1050 to $1600 \mathrm{~g} \cdot \mathrm{ai} \cdot \mathrm{ha}^{-1}$ and the halosulfuron label has a dose range of 25 to $50 \mathrm{~g} \cdot \mathrm{ai} \cdot \mathrm{ha}^{-1}$ Earlier research has primarily focused on halosulfuron at $35 \mathrm{~g} \cdot \mathrm{ai} \cdot \mathrm{ha}^{-1}$ for weed control in white bean [6] [9] [10] [16]. Limited information exists on the effect of $S$-metolachlor plus lower doses of halosulfuron particularly at the lowest labelled dose of $25 \mathrm{~g} \cdot \mathrm{ai} \cdot \mathrm{ha}^{-1}$ for weed control in white bean. Studies are needed to determine the appropriate ap- 
plication dose of halosulfuron alone or in tankmix with $S$-metolachlor for broad and comprehensive weed control in white bean. This information will allow producers to reduce their input costs and minimize crop losses from weed interference in white bean.

The purpose of this research was to evaluate how doses of $S$-metolachlor and halosulfuron should be adjusted to control specific problematic weeds in white bean production.

\section{Materials and Methods}

Field experiments (total of 5) were established at the University of Guelph Research Station near Exeter (43 $\left.19^{\prime} 1.2108^{\prime \prime N}, 81^{\circ} 30^{\prime} 3.8736^{\prime \prime E}\right)$ in 2016 and 2017 and at the University of Guelph Ridgetown Campus near Ridgetown $\left(42^{\circ} 26^{\prime} 41.46^{\prime \prime} \mathrm{N}\right.$, $81^{\circ} 52^{\prime} 44.472 \mathrm{~W}$ ) during 2016 to 2018 . The experimental design was a randomized complete block design (RCBD) with 4 replications. Treatments included a weedy control, weed-free control, $S$-metolachlor at 1050 and $1600 \mathrm{~g} \cdot \mathrm{ai} \cdot \mathrm{ha}^{-1}$, halosulfuron at $25,37.5$ and $50 \mathrm{~g} \cdot \mathrm{ai} \cdot \mathrm{ha}^{-1}, S$-metolachlor at $1050 \mathrm{~g} \cdot \mathrm{ai} \cdot \mathrm{ha} \mathrm{a}^{-1}+$ halosulfuron at $25,37.5$ or $50 \mathrm{~g} \cdot \mathrm{ai} \cdot \mathrm{ha}^{-1}$, and $S$-metolachlor at $1600 \mathrm{~g} \cdot \mathrm{ai} \cdot \mathrm{ha}^{-1}+$ halosulfuron at $25,37.5$ or $50 \mathrm{~g} \cdot \mathrm{ai} \cdot \mathrm{ha}^{-1}$. Plots within each experiment included four rows of white bean (“T9905") spaced $75 \mathrm{~cm}$ apart and were $8 \mathrm{~m}$ long at Ridgetown and $10 \mathrm{~m}$ long at Exeter. White bean was seeded 3.5 to $4.5 \mathrm{~cm}$ deep at a rate of approximately 240,000 seeds $^{-1}$ in late May to early June of each year.

Herbicides were sprayed preemergence (PRE) one to two days after seeding with a backpack sprayer which was pressurized with $\mathrm{CO}_{2}$ and was calibrated to deliver $200 \mathrm{~L} \cdot \mathrm{ha}^{-1}$ of water at $240 \mathrm{kPa}$.

Injury in white bean was assessed visually 2 and 4 weeks after white bean emergence (WAE) and weed control assessments was made 4 and $8 \mathrm{WAE}$ based on a rating of 0 to 100 where 0 represented no injury or weed control and 100 represented total bean or weed necrosis. Weed density (counts) and weed shoot dry weight (biomass) were evaluated 8 WAE by harvesting weeds from two $0.25 \mathrm{~m}^{-2}$ quadrats (counted and dried at $60^{\circ} \mathrm{C}$ in a paper bag for at least 72 hours) within each experimental plot. White bean in each experimental plot was harvested during September/October of each year.

The GLIMMIX procedure in SAS [17] was used to analyze the data. In the analysis, herbicide treatment was the fixed effect and environment (year-location combinations), replicate within the environment and the environment-treatment interaction were the random effects. The best distribution and associated link function for each parameter was chosen by comparing fit statistics, residual plots and the Shapiro-Wilk statistic among the potential distributions. LSMEANS were calculated by using the inverse link function, and pairwise comparisons were subjected to Tukey's adjustment before determining treatment differences at $\mathrm{P}<0.05$. The Gaussian distribution and identity link were used for percent visible white bean injury 2 and $4 \mathrm{WAE}$, percent visible weed control of $A$. theophrasti and C. album $8 \mathrm{WAE}, E$. crus-galli dry weight and white bean yield. 
Percent visible weed control of all remaining weed species at 2 and 4 WAE were analyzed using arcsine square root distribution and identity link. Weed density and weed shoot dry weight were analyzed using the lognormal distribution and identity link. The weedy control (assigned a value of 0 for injury and weed control) and weed-free control (assigned a value of 0 for injury, weed density and biomass, or 100 for weed control) were excluded from the analysis due to zero variance. Comparisons were still possible between the other treatments and the value zero using the LSMEANS output and differences were identified. Arcsine square root and lognormal distributions were back-transformed for presentation of results.

\section{Results and Discussion}

\subsection{White Bean Injury and Yield}

Visible white bean injury from the herbicides evaluated was minimal. $S$-metolachlor, halosulfuron, and $S$-metolachlor + halosulfuron, applied PRE, caused < $5 \%$ injury in white bean 2 and 4 WAE (Table 1 ). The level of injury is consistent with other research that have shown minimal, and transient, injury in white bean with $S$-metolachlor and halosulfuron [6] [9] [10] [16].

Weed interference delayed maturity (as indicated by seed moisture content at harvest) and reduced white bean seed yield 54\%. Interference from weeds with

Table 1. Visible injury 2 and 4 WAE, percent moisture at maturity and yield of white bean treated with $S$-metolachlor and halosulfuron applied PRE at Exeter and Ridgetown $(2016-2018)^{\mathrm{a}, \mathrm{b}}$

\begin{tabular}{|c|c|c|c|c|c|}
\hline \multirow{2}{*}{ Treatment } & \multirow{2}{*}{$\begin{array}{c}\text { Dose } \\
\left(\mathrm{g} \cdot \mathrm{ai}^{\circ} \mathrm{ha}^{-1}\right)\end{array}$} & \multicolumn{2}{|c|}{ Injury (\%) } & \multirow{2}{*}{$\begin{array}{c}\text { Seed } \\
\text { Moisture }\end{array}$} & \multirow{2}{*}{$\begin{array}{c}\text { Yield } \\
\left(\mathrm{T} \cdot \mathrm{ha}^{-1}\right)\end{array}$} \\
\hline & & $2 \mathrm{WAE}$ & $4 \mathrm{WAE}$ & & \\
\hline Weedy control & & $0^{\mathrm{a}}$ & $0^{\mathrm{a}}$ & $19.48^{\mathrm{d}}$ & $1.1^{\mathrm{e}}$ \\
\hline Weed-free control & & $0^{\mathrm{a}}$ & $0^{\mathrm{a}}$ & $18.02^{\mathrm{a}}$ & $2.4^{\mathrm{a}}$ \\
\hline$S$-metolachlor & 1050 & $2^{\mathrm{b}}$ & $2^{\mathrm{a}}$ & $19.23^{\mathrm{bcd}}$ & $1.3^{\mathrm{de}}$ \\
\hline$S$-metolachlor & 1600 & $3^{\mathrm{b}}$ & $4^{\mathrm{a}}$ & $19.39^{\mathrm{cd}}$ & $1.4^{\mathrm{de}}$ \\
\hline Halosulfuron & 25 & $1^{a} b$ & $1^{\mathrm{a}}$ & $18.79^{\mathrm{abcd}}$ & $1.6^{\mathrm{cd}}$ \\
\hline Halosulfuron & 37.5 & $2^{\mathrm{b}}$ & $2^{\mathrm{a}}$ & $18.38^{\mathrm{a}}$ & $1.8^{\mathrm{bcd}}$ \\
\hline Halosulfuron & 50 & $2^{\mathrm{b}}$ & $3^{\mathrm{a}}$ & $18.53^{\mathrm{ab}}$ & $1.7^{\mathrm{bcd}}$ \\
\hline$S$-metolachlor + halosulfuron & $1050+25$ & $2^{\mathrm{b}}$ & $2^{\mathrm{a}}$ & $18.57^{\mathrm{abc}}$ & $2.2^{\mathrm{ab}}$ \\
\hline$S$-metolachlor + halosulfuron & $1050+37.5$ & $3^{\mathrm{b}}$ & $3^{\mathrm{a}}$ & $18.34^{\mathrm{a}}$ & $2.1^{\mathrm{abc}}$ \\
\hline$S$-metolachlor + halosulfuron & $1050+50$ & $4^{\mathrm{b}}$ & $4^{\mathrm{a}}$ & $18.35^{\mathrm{a}}$ & $2.1^{\mathrm{abc}}$ \\
\hline$S$-metolachlor + halosulfuron & $1600+25$ & $3^{\mathrm{b}}$ & $3^{\mathrm{a}}$ & $18.55^{\mathrm{ab}}$ & $2.1^{\mathrm{abc}}$ \\
\hline$S$-metolachlor + halosulfuron & $1600+37.5$ & $4^{\mathrm{b}}$ & $4^{\mathrm{a}}$ & $18.30^{\mathrm{a}}$ & $2.1^{\mathrm{abc}}$ \\
\hline$S$-metolachlor + halosulfuron & $1600+50$ & $4^{\mathrm{b}}$ & $3^{\mathrm{a}}$ & $18.15^{\mathrm{a}}$ & $2.0^{\mathrm{abc}}$ \\
\hline
\end{tabular}

${ }^{a}$ Abbreviations: PRE, preemergence; WAE, weeks after white bean emergence. ${ }^{b}$ Means followed by a different letter within a column are significantly different according to a Tukey-Kramer multiple range test at $\mathrm{P}<$ 0.05 . 
$S$-metolachlor and halosulfuron applied alone reduced white bean seed yield as much as $46 \%$ and $33 \%$, respectively (Table 1 ). White bean seed yield with the $S$-metolachlor + halosulfuron tankmixes at all doses evaluated was similar to the weed-free control. Results are consistent with other studies that have shown minimal crop injury in white bean with $S$-metholachlor (1600 g.ai $\left.\cdot \mathrm{ha}^{-1}\right)$, halosulfuron $\left(35\right.$ g.ai·ha $\left.{ }^{-1}\right)$, and $S$-metolachlor + halosulfuron $\left(1050+35\right.$ g.ai.ha $\left.{ }^{-1}\right)$ [6] [7] [9] [10].

\subsection{Weed Control}

Weeds selected for analysis needed to be present in at least 2 out of the 5 environments. Major weed species present on study sites included $A$. theophrasti, $A$. retroflexus, C. album, $A$. artemisiifolia, E. crus-galli and $S$. viridis.

\subsubsection{Abutilon theophrasti}

$S$-metolachlor at doses evaluated controlled $A$. theophrasti $\leq 3 \%$ (Table 2). Halosulfuron at the doses evaluated controlled A. theophrasti 39\% to $87 \%$. $S$-metolachlor (1050 g.ai $\left.\cdot \mathrm{ha}^{-1}\right)+$ halosulfuron at $25,37.5$ and $50 \mathrm{~g} \cdot \mathrm{ai} \cdot \mathrm{ha}^{-1}$ provided as much as $64 \%, 78 \%$ and $89 \%$ control of $A$. theophrasti, respectively. $S$-metolachlor (1600 g.ai $\left.\cdot \mathrm{ha}^{-1}\right)+$ halosulfuron at $25,37.5$ and $50 \mathrm{~g} \cdot \mathrm{ai} \cdot \mathrm{ha}^{-1}$ provided as much as $80 \%, 88 \%$ and $94 \%$ control of $A$. theophrasti, respectively. All herbicide treatments resulted in $A$. theophrasti density and shoot dry weight that was comparable to the weedy control (Table 2).

Table 2. Percent visible control 4 and 8 WAE, density and dry weight of Abutilon theophrasti treated with $S$-metolachlor and halosulfuron applied PRE at Ridgetown (2016$2018)^{\mathrm{a}, \mathrm{b}}$

\begin{tabular}{|c|c|c|c|c|c|}
\hline \multirow{2}{*}{ Treatment } & \multirow{2}{*}{$\begin{array}{c}\text { Dose } \\
\left(\mathrm{g} \cdot \mathrm{ai} \cdot \mathrm{ha}^{-1}\right)\end{array}$} & \multicolumn{2}{|c|}{ Control (\%) } & \multirow{2}{*}{$\begin{array}{l}\text { Density } \\
\left(\text { no. } \mathrm{m}^{-2}\right)\end{array}$} & \multirow{2}{*}{$\begin{array}{c}\text { Dry weight } \\
\left(\mathrm{g} \cdot \mathrm{m}^{-2}\right)\end{array}$} \\
\hline & & $4 \mathrm{WAE}$ & $8 \mathrm{WAE}$ & & \\
\hline Weedy control & & $0^{c}$ & $0^{\mathrm{d}}$ & $7.4^{\mathrm{b}}$ & $6.0^{\mathrm{b}}$ \\
\hline Weed-free control & & 100 & 100 & $0.0^{\mathrm{a}}$ & $0.0^{\mathrm{a}}$ \\
\hline$S$-metolachlor & 1050 & $1^{\mathrm{c}}$ & $0^{\mathrm{d}}$ & $5.8^{\mathrm{b}}$ & $7.6^{\mathrm{b}}$ \\
\hline$S$-metolachlor & 1600 & $3^{c}$ & $2^{\mathrm{d}}$ & $5.1^{\mathrm{b}}$ & $8.2^{\mathrm{b}}$ \\
\hline Halosulfuron & 25 & $57^{\mathrm{b}}$ & $39^{c}$ & $3.0^{\mathrm{b}}$ & $2.7^{\mathrm{b}}$ \\
\hline Halosulfuron & 37.5 & $74^{\mathrm{ab}}$ & $61^{\mathrm{abc}}$ & $4.2^{\mathrm{b}}$ & $2.7^{\mathrm{b}}$ \\
\hline Halosulfuron & 50 & $87^{\mathrm{ab}}$ & $74^{\mathrm{ab}}$ & $3.0^{\mathrm{b}}$ & $1.7^{\mathrm{b}}$ \\
\hline$S$-metolachlor + halosulfuron & $1050+25$ & $64^{\mathrm{b}}$ & $47^{\mathrm{c}}$ & $3.9^{\mathrm{b}}$ & $3.1^{\mathrm{b}}$ \\
\hline$S$-metolachlor + halosulfuron & $1050+37.5$ & $78^{\mathrm{ab}}$ & $61^{\mathrm{abc}}$ & $3.9^{\mathrm{b}}$ & $4.0^{\mathrm{b}}$ \\
\hline$S$-metolachlor + halosulfuron & $1050+50$ & $89^{\mathrm{ab}}$ & $79^{\mathrm{ab}}$ & $3.0^{\mathrm{b}}$ & $1.9^{\mathrm{b}}$ \\
\hline$S$-metolachlor + halosulfuron & $1600+25$ & $80^{\mathrm{ab}}$ & $59^{\mathrm{bc}}$ & $2.2^{\mathrm{b}}$ & $2.0^{\mathrm{b}}$ \\
\hline$S$-metolachlor + halosulfuron & $1600+37.5$ & $88^{\mathrm{ab}}$ & $76^{\mathrm{ab}}$ & $3.2^{\mathrm{b}}$ & $3.6^{\mathrm{b}}$ \\
\hline$S$-metolachlor + halosulfuron & $1600+50$ & $94^{\mathrm{a}}$ & $86^{\mathrm{a}}$ & $2.4^{\mathrm{b}}$ & $1.1^{\mathrm{ab}}$ \\
\hline
\end{tabular}

${ }^{a}$ Abbreviations: PRE, preemergence; WAE, weeks after white bean emergence. ${ }^{b}$ Means followed by a different letter within a column are significantly different according to a Tukey-Kramer multiple range test at $\mathrm{P}<$ 0.05 . 


\subsubsection{Amaranthus retroflexus}

$S$-metolachlor and halosulfuron applied alone at doses evaluated controlled $A$. retroflexus $78 \%$ to $93 \%$ and $93 \%$ to $99 \%$, respectively (Table 3). $S$-metolachlor $\left(1050\right.$ or $\left.1600 \mathrm{~g} \cdot \mathrm{ai} \cdot \mathrm{ha}^{-1}\right)+$ halosulfuron at $25,37.5$ and $50 \mathrm{~g} \cdot \mathrm{ai} \cdot \mathrm{ha}^{-1}$ provided excellent (98\% to $100 \%)$ control of $A$. retroflexus. Increasing the dose of $S$-metolachlor or halosulfuron did not significantly increase $A$. retroflexus control.

A. retroflexus density and dry weight reductions with herbicides evaluated were consistent with the visible control assessments (Table 3). $S$-metolachlor, halosulfuron, and $S$-metolachlor + halosulfuron reduced $A$. retroflexus density as much as $87 \%, 97 \%$ and $98 \%$ and $A$. retroflexus dry weight as much as $95 \%$, $99 \%$ and $100 \%$, respectively (Table 3 ).

Other studies have similarly shown $84 \%$ to $95 \%$ control of $A$. retroflexus with $S$-metolachlor and $83 \%$ to $100 \%$ control of $A$. retroflexus with halosulfuron in white bean [7] [9]. Brown and Masiunas [19] also reported 94\% and 98\% A. retroflexus control with halosulfuron at 3 and 6 weeks after application (WAA), respectively. Other studies have also reported as much as $96 \%$ to $100 \%$ A. retroflexus control with $S$-metolachlor and halosufuron tankmix in white bean [6] [7] [9] [18]. Li et al. [7] found 100\% A. retroflexus control in white bean with $S$-metolachlor + halosulfuron at $1050+35 \mathrm{~g} \cdot \mathrm{ai} \cdot \mathrm{ha}^{-1}$.

Table 3. Percent visible control 4 and $8 \mathrm{WAE}$, density and dry weight of Amaranthus retroflexus treated with $S$-metolachlor and halosulfuron applied PRE at Exeter (20162017) and Ridgetown (2017).

\begin{tabular}{|c|c|c|c|c|c|}
\hline \multirow{2}{*}{ Treatment } & \multirow{2}{*}{$\begin{array}{c}\text { Dose } \\
\left(\mathrm{g} \cdot \mathrm{ai} \cdot \mathrm{ha}^{-1}\right)\end{array}$} & \multicolumn{2}{|c|}{ Control } & \multirow{2}{*}{$\begin{array}{l}\text { Density } \\
\left(\text { no. } \mathrm{m}^{-2} \text { ) }\right.\end{array}$} & \multirow{2}{*}{$\begin{array}{c}\text { Dry weight } \\
\left(\mathrm{g} \cdot \mathrm{m}^{-2}\right)\end{array}$} \\
\hline & & $4 \mathrm{WAE}$ & 8 WAE & & \\
\hline Weedy control & & $0^{c}$ & $0^{c}$ & $28.6^{c}$ & $36.0^{\mathrm{d}}$ \\
\hline Weed-free control & & 100 & 100 & $0^{\mathrm{a}}$ & $0^{\mathrm{a}}$ \\
\hline$S$-metolachlor & 1050 & $81.9^{\mathrm{b}}$ & $78.4^{\mathrm{b}}$ & $4.0^{\mathrm{b}}$ & $2.3^{\mathrm{c}}$ \\
\hline$S$-metolachlor & 1600 & $92.9^{\mathrm{ab}}$ & $92.7^{\mathrm{ab}}$ & $3.7^{\mathrm{b}}$ & $1.7^{\mathrm{bc}}$ \\
\hline Halosulfuron & 25 & $95.2^{\mathrm{ab}}$ & $95.2^{\mathrm{ab}}$ & $1.7^{\mathrm{ab}}$ & $0.6^{\mathrm{abc}}$ \\
\hline Halosulfuron & 37.5 & $95.9^{\mathrm{ab}}$ & $92.6^{\mathrm{ab}}$ & $2.5^{\mathrm{ab}}$ & $0.9^{\mathrm{abc}}$ \\
\hline Halosulfuron & 50 & $98.8^{\mathrm{ab}}$ & $97.9^{\mathrm{a}}$ & $0.9^{\mathrm{ab}}$ & $0.4^{\mathrm{abc}}$ \\
\hline$S$-metolachlor + halosulfuron & $1050+25$ & $98.9^{\mathrm{ab}}$ & $97.9^{\mathrm{a}}$ & $1.8^{\mathrm{ab}}$ & $0.6^{\mathrm{abc}}$ \\
\hline$S$-metolachlor + halosulfuron & $1050+37.5$ & $99.2^{\mathrm{a}}$ & $99.3^{\mathrm{a}}$ & $1.4^{\mathrm{ab}}$ & $0.3^{\mathrm{abc}}$ \\
\hline$S$-metolachlor + halosulfuron & $1050+50$ & $98.9^{\mathrm{ab}}$ & $98.7^{\mathrm{a}}$ & $0.8^{\mathrm{ab}}$ & $0.5^{\mathrm{abc}}$ \\
\hline$S$-metolachlor + halosulfuron & $1600+25$ & $99.2^{\mathrm{a}}$ & $98.3^{\mathrm{a}}$ & $1.1^{\mathrm{ab}}$ & $0.4^{\mathrm{abc}}$ \\
\hline$S$-metolachlor + halosulfuron & $1600+37.5$ & $99.6^{\mathrm{a}}$ & $99.6^{\mathrm{a}}$ & $1.9^{\mathrm{ab}}$ & $0.7^{\mathrm{abc}}$ \\
\hline$S$-metolachlor + halosulfuron & $1600+50$ & $99.7^{\mathrm{a}}$ & $99.8^{\mathrm{a}}$ & $0.6^{\mathrm{ab}}$ & $0.1^{\mathrm{abc}}$ \\
\hline
\end{tabular}

${ }^{a}$ Abbreviations: PRE, preemergence; WAE, weeks after white bean emergence. ${ }^{b}$ Means followed by a different letter within a column are significantly different according to a Tukey-Kramer multiple range test at $\mathrm{P}<$ 0.05 . 


\subsubsection{Ambrosia artemisiifolia}

$S$-metolachlor alone at doses evaluated provided only $0 \%$ to $9 \%$ control of $A$. artemisiifolia (Table 4). However, halosulfuron alone at doses evaluated controlled A. artemisiifolia $64 \%$ to $88 \%$. $S$-metolachlor $\left(1050 \mathrm{~g} \cdot \mathrm{ai} \cdot \mathrm{ha}^{-1}\right)+$ halosulfuron at 25 , 37.5 and 50 g.ai $\cdot \mathrm{ha}^{-1}$ controlled $A$. artemisiifolia $78 \%$ to $91 \%$. Similarly, $S$-metolachlor (1600 g.ai ha $\left.{ }^{-1}\right)+$ halosulfuron at 25, 37.5 and $50 \mathrm{~g} \cdot \mathrm{ai} \cdot \mathrm{ha}^{-1}$ provided $83 \%$ to $94 \%$ A. artemisiifolia control.

$S$-metolachlor provided no reduction in density or dry weight of $A$. artemisiifolia at the doses evaluated (Table 4). However, halosulfuron and $S$-metolachlor + halosulfuron treatments reduced $A$. artemisiifolia density or dry weight as much as $95 \%$ (Table 4 ).

Other research has shown only $13 \%$ to $40 \%$ control of $A$. artemisiifolia with $S$-metolachlor and $95 \%$ to $99 \%$ control of $A$. artemisiifolia with halosulfuron in white bean [7] [9]. Li et al. [7] reported 95\% to 98\% A. artemisiifolia control in white bean with $S$-metolachlor + halosulfuron at $1050+35 \mathrm{~g} \cdot \mathrm{ai} \cdot \mathrm{ha}^{-1}$.

\subsubsection{Chenopodium album}

$S$-metolachlor applied alone at the doses evaluated provided poor (5\% to $15 \%$ ) control of $C$. album (Table 5). Halosulfuron alone at doses evaluated controlled C. album only $34 \%$ to $59 \%$. $S$-metolachlor + halosulfuron at doses evaluated also provided less than adequate control (37\% to $78 \%)$ of $C$. album. Increasing the

Table 4. Percent visible control 4 and $8 \mathrm{WAE}$, density and dry weight of Ambrosia artemisiifolia treated with $S$-metolachlor and halosulfuron applied PRE at Exeter (2017) and Ridgetown (2016-2018) $)^{\mathrm{a}, \mathrm{b}}$.

\begin{tabular}{|c|c|c|c|c|c|}
\hline \multirow{2}{*}{ Treatment } & \multirow{2}{*}{$\begin{array}{c}\text { Dose } \\
\left(\mathrm{g} \cdot \mathrm{ai} \cdot \mathrm{ha}^{-1}\right)\end{array}$} & \multicolumn{2}{|c|}{ Control (\%) } & \multirow{2}{*}{$\begin{array}{c}\text { Density } \\
\left(\text { no. } \mathrm{m}^{-2}\right)\end{array}$} & \multirow{2}{*}{$\begin{array}{c}\text { Dry weight } \\
\left(\mathrm{g} \cdot \mathrm{m}^{-2}\right)\end{array}$} \\
\hline & & $4 \mathrm{WAE}$ & $8 \mathrm{WAE}$ & & \\
\hline Weedy control & & $0^{c}$ & $0^{\mathrm{d}}$ & $43.1^{\mathrm{c}}$ & $50.2^{\mathrm{c}}$ \\
\hline Weed-free control & & 100 & 100 & $0^{\mathrm{a}}$ & $0^{\mathrm{a}}$ \\
\hline$S$-metolachlor & 1050 & $7^{\mathrm{b}}$ & $0^{\mathrm{d}}$ & $33.7^{\mathrm{c}}$ & $63.1^{c}$ \\
\hline$S$-metolachlor & 1600 & $9^{\mathrm{b}}$ & $0^{\mathrm{d}}$ & $27.8^{\mathrm{c}}$ & $51.0^{c}$ \\
\hline Halosulfuron & 25 & $78^{\mathrm{a}}$ & $64^{c}$ & $5.9^{\mathrm{b}}$ & $7.3^{\mathrm{b}}$ \\
\hline Halosulfuron & 37.5 & $81^{\mathrm{a}}$ & $72^{\mathrm{bc}}$ & $4.6^{\mathrm{b}}$ & $6.5^{\mathrm{b}}$ \\
\hline Halosulfuron & 50 & $88^{\mathrm{a}}$ & $81^{\mathrm{abc}}$ & $4.5^{\mathrm{b}}$ & $4.9^{\mathrm{b}}$ \\
\hline$S$-metolachlor + halosulfuron & $1050+25$ & $86^{\mathrm{a}}$ & $78^{\mathrm{abc}}$ & $5.6^{\mathrm{b}}$ & $6.0^{\mathrm{b}}$ \\
\hline$S$-metolachlor + halosulfuron & $1050+37.5$ & $91^{\mathrm{a}}$ & $84^{\mathrm{ab}}$ & $5.5^{\mathrm{b}}$ & $8.0^{\mathrm{b}}$ \\
\hline$S$-metolachlor + halosulfuron & $1050+50$ & $91^{\mathrm{a}}$ & $85^{\mathrm{ab}}$ & $3.3^{\mathrm{b}}$ & $5.4^{\mathrm{b}}$ \\
\hline$S$-metolachlor + halosulfuron & $1600+25$ & $89^{\mathrm{a}}$ & $83^{\mathrm{abc}}$ & $3.5^{\mathrm{b}}$ & $6.1^{\mathrm{b}}$ \\
\hline$S$-metolachlor + halosulfuron & $1600+37.5$ & $93^{\mathrm{a}}$ & $89^{\mathrm{a}}$ & $2.8^{\mathrm{b}}$ & $5.2^{\mathrm{b}}$ \\
\hline$S$-metolachlor + halosulfuron & $1600+50$ & $94^{\mathrm{a}}$ & $88^{\mathrm{ab}}$ & $2.2^{\mathrm{b}}$ & $2.5^{\mathrm{b}}$ \\
\hline
\end{tabular}

${ }^{a}$ Abbreviations: PRE, preemergence; WAE, weeks after white bean emergence. ${ }^{b}$ Means followed by a different letter within a column are significantly different according to a Tukey-Kramer multiple range test at $\mathrm{P}<$ 0.05 . 
Table 5. Percent visible control 4 and $8 \mathrm{WAE}$, density and dry weight of Chenopodium albumtreated with $S$-metolachlor and halosulfuron applied PRE at Exeter and Ridgetown $(2016-2018)^{\mathrm{a}, \mathrm{b}}$.

\begin{tabular}{|c|c|c|c|c|c|}
\hline \multirow{2}{*}{ Treatment } & \multirow{2}{*}{$\begin{array}{c}\text { Dose } \\
\left(\mathrm{g} \cdot \mathrm{ai} \cdot \mathrm{ha}^{-1}\right)\end{array}$} & \multicolumn{2}{|c|}{ Control (\%) } & \multirow{2}{*}{$\begin{array}{l}\text { Density } \\
\left(\text { no. } \mathrm{m}^{-2}\right)\end{array}$} & \multirow{2}{*}{$\begin{array}{c}\text { Dry weight } \\
\left(\mathrm{g} \cdot \mathrm{m}^{-2}\right)\end{array}$} \\
\hline & & $4 \mathrm{WAE}$ & $8 \mathrm{WAE}$ & & \\
\hline Weedy control & & $0^{c}$ & $0^{\mathrm{d}}$ & $30.9^{\mathrm{f}}$ & $13.2^{\mathrm{c}}$ \\
\hline Weed-free control & & 100 & 100 & $0^{\mathrm{a}}$ & $0^{\mathrm{a}}$ \\
\hline$S$-metolachlor & 1050 & $5^{c}$ & $14^{\mathrm{cd}}$ & $10.5^{\mathrm{e}}$ & $10.7^{\mathrm{bc}}$ \\
\hline$S$-metolachlor & 1600 & $6^{c}$ & $15^{\mathrm{cd}}$ & $8.2^{\mathrm{de}}$ & $10.4^{\mathrm{bc}}$ \\
\hline Halosulfuron & 25 & $35^{\mathrm{b}}$ & $34^{\mathrm{bc}}$ & $3.2^{\mathrm{bc}}$ & $3.7^{\mathrm{bc}}$ \\
\hline Halosulfuron & 37.5 & $49^{\mathrm{ab}}$ & $45^{\mathrm{ab}}$ & $3.3^{\mathrm{cd}}$ & $2.7^{\mathrm{bc}}$ \\
\hline Halosulfuron & 50 & $59^{\mathrm{ab}}$ & $48^{\mathrm{ab}}$ & $2.0^{\mathrm{bc}}$ & $3.5^{\mathrm{bc}}$ \\
\hline$S$-metolachlor + halosulfuron & $1050+25$ & $47^{\mathrm{ab}}$ & $37^{\mathrm{abc}}$ & $2.7^{b c}$ & $4.1^{\mathrm{bc}}$ \\
\hline$S$-metolachlor + halosulfuron & $1050+37.5$ & $54^{\mathrm{ab}}$ & $48^{\mathrm{ab}}$ & $1.9^{\mathrm{bc}}$ & $2.4^{\mathrm{bc}}$ \\
\hline$S$-metolachlor + halosulfuron & $1050+50$ & $64^{\mathrm{ab}}$ & $61^{\mathrm{a}}$ & $1.0^{\mathrm{bc}}$ & $2.8^{\mathrm{bc}}$ \\
\hline $\mathcal{S}$-metolachlor + halosulfuron & $1600+25$ & $66^{\mathrm{ab}}$ & $46^{\mathrm{a} b}$ & $2.1^{\mathrm{bc}}$ & $5.3^{\mathrm{bc}}$ \\
\hline$S$-metolachlor + halosulfuron & $1600+37.5$ & $71^{\mathrm{a}}$ & $62^{\mathrm{a}}$ & $1.0^{\mathrm{b}}$ & $1.8^{\mathrm{b}}$ \\
\hline$S$-metolachlor + halosulfuron & $1600+50$ & $78^{\mathrm{a}}$ & $61^{\mathrm{a}}$ & $1.2^{\mathrm{bc}}$ & $2.0^{\mathrm{b}}$ \\
\hline
\end{tabular}

${ }^{a}$ Abbreviations: PRE, preemergence; WAE, weeks after white bean emergence. ${ }^{b}$ Means followed by a different letter within a column are significantly different according to a Tukey-Kramer multiple range test at $\mathrm{P}<$ 0.05 .

dose of $S$-metolachlor or halosulfuron did not significantly increase the control of C. album.

$S$-metolachlor, halosulfuron, and $S$-metolachlor + halosulfuron reduced $C$. album density as much as $73 \%, 94 \%$ and $97 \%$, respectively. However, shoot weight was not different than the weedy control with all herbicide treatments except for $S$-metolachlor $\left(1600 \mathrm{~g} \cdot \mathrm{ai} \cdot \mathrm{ha}^{-1}\right)+$ halosulfuron at 37.5 and $50 \mathrm{~g} \cdot \mathrm{ai} \cdot \mathrm{ha}^{-1}$ which reduced $C$. album dry weight $86 \%$ and $85 \%$, respectively (Table 5).

In other research, $S$-metolachlor applied alone provided $19 \%$ to $82 \%$ C. album control in white bean [7] [9]. Brown and Masiunas [19] reported $90 \%$ to $98 \% C$. album control with halosulfuron at 3 to 6 WAA. Other studies have also reported $96 \%$ to $100 \%$ C. album control with halosulfuron in white bean [7] [9]. Li et al. [7] reported $99 \%$ to $100 \%$ C. album control with $S$-metolachlor + halosulfuron at $1050+35$ g.aihha ${ }^{-1}$.

\subsubsection{Echinochloa crus-galli}

All treatments that included $S$-metolachlor provided excellent $E$. crus-galli control (Table 6). $S$-metolachlor applied alone at the doses evaluated controlled $E$. crus-galli $97 \%$ to $99 \%$ (Table 6). In contrast, halosulfuron applied at 25, 37.5 and $50 \mathrm{~g} \cdot \mathrm{ai} \cdot \mathrm{ha}^{-1}$ controlled E. crus-galli only $10 \%$ to $30 \%$ in white bean (Table 6). $S$-metolachlor (1050 g.ai $\left.\cdot \mathrm{ha}^{-1}\right)+$ halosulfuron at $25,37.5$ and $50 \mathrm{~g} \cdot \mathrm{ai}^{\mathrm{h}} \mathrm{ha}^{-1}$ 
Table 6. Percent visible control 4 and 8 WAE, density and dry weight of Echinochloa crus-galli treated with $S$-metolachlor and halosulfuron applied PRE at Exeter (2017) and Ridgetown $(2018)^{\mathrm{a}, \mathrm{b}}$.

\begin{tabular}{|c|c|c|c|c|c|}
\hline \multirow{2}{*}{ Treatment } & \multirow{2}{*}{$\begin{array}{c}\text { Dose } \\
\left(\mathrm{g} \cdot \mathrm{ai} \cdot \mathrm{ha}^{-1}\right)\end{array}$} & \multicolumn{2}{|c|}{ Control (\%) } & \multirow{2}{*}{$\begin{array}{c}\text { Density } \\
\left(\text { no. } \mathrm{m}^{-2}\right)\end{array}$} & \multirow{2}{*}{$\begin{array}{c}\text { Dry weight } \\
\left(\mathrm{g} \cdot \mathrm{m}^{-2}\right)\end{array}$} \\
\hline & & $4 \mathrm{WAE}$ & $8 \mathrm{WAE}$ & & \\
\hline Weedy control & & $0^{c}$ & $0^{c}$ & $21.8^{\mathrm{d}}$ & $26.3^{\mathrm{bc}}$ \\
\hline Weed-free control & & 100 & 100 & $0 \mathrm{a}$ & $0^{\mathrm{a}}$ \\
\hline$S$-metolachlor & 1050 & $97^{\mathrm{a}}$ & $98^{\mathrm{a}}$ & $2.5^{\mathrm{b}}$ & $1.1^{\mathrm{b}}$ \\
\hline$S$-metolachlor & 1600 & $99^{\mathrm{a}}$ & $99^{\mathrm{a}}$ & $2.2^{\mathrm{b}}$ & $1.0^{\mathrm{b}}$ \\
\hline Halosulfuron & 25 & $26^{\mathrm{b}}$ & $21^{\mathrm{b}}$ & $28.2^{\mathrm{d}}$ & $39.2^{\mathrm{c}}$ \\
\hline Halosulfuron & 37.5 & $28^{\mathrm{b}}$ & $25^{\mathrm{b}}$ & $15.1^{\mathrm{cd}}$ & $28.0^{\mathrm{bc}}$ \\
\hline Halosulfuron & 50 & $30^{\mathrm{b}}$ & $10^{\mathrm{b}}$ & $27.5^{\mathrm{d}}$ & $23.7^{\mathrm{bc}}$ \\
\hline$S$-metolachlor + halosulfuron & $1050+25$ & $94^{\mathrm{a}}$ & $97^{\mathrm{a}}$ & $4.0^{\mathrm{bc}}$ & $3.5^{\mathrm{b}}$ \\
\hline$S$-metolachlor + halosulfuron & $1050+37.5$ & $97^{\mathrm{a}}$ & $98^{\mathrm{a}}$ & $3.6^{\mathrm{bc}}$ & $1.8^{\mathrm{b}}$ \\
\hline $\mathcal{S}$-metolachlor + halosulfuron & $1050+50$ & $94^{\mathrm{a}}$ & $96^{\mathrm{a}}$ & $5.0^{\mathrm{bc}}$ & $2.1^{\mathrm{b}}$ \\
\hline$S$-metolachlor + halosulfuron & $1600+25$ & $98^{\mathrm{a}}$ & $98^{\mathrm{a}}$ & $2.7^{\mathrm{b}}$ & $1.0^{\mathrm{b}}$ \\
\hline$S$-metolachlor + halosulfuron & $1600+37.5$ & $95^{\mathrm{a}}$ & $98^{\mathrm{a}}$ & $2.7^{\mathrm{b}}$ & $0.9^{\mathrm{b}}$ \\
\hline$S$-metolachlor + halosulfuron & $1600+50$ & $98^{\mathrm{a}}$ & $98^{\mathrm{a}}$ & $2.4^{\mathrm{b}}$ & $10.4^{\mathrm{bc}}$ \\
\hline
\end{tabular}

${ }^{a}$ Abbreviations: PRE, preemergence; WAE, weeks after white bean emergence. ${ }^{b}$ Means followed by a different letter within a column are significantly different according to a Tukey-Kramer multiple range test at $\mathrm{P}<$ 0.05 .

provided $97 \%, 98 \%$ and $96 \%$ control of E. crus-galli in white bean, respectively 8 WAE. Similarly, $S$-metolachlor $\left(1600 \mathrm{~g} \cdot \mathrm{ai} \cdot \mathrm{ha}^{-1}\right)+$ halosulfuron at $25,37.5$, and 50 g.ai.ha ${ }^{-1}$ controlled E. crus-galli as much as $98 \%$ in white bean.

$S$-metolachlor and $S$-metolachlor + halosulfuron reduced density of E. crusgalli as much as $90 \%$ and $89 \%$, respectively. However, E. crus-galli density and shoot dry weight was not different than the weedy control with halosulfuron (Table 6).

\subsubsection{Setaria viridis}

All treatments that included $S$-metolachlor provided excellent $S$. viridis control (Table 7). $S$-metolachlor applied alone at the doses evaluated provided $96 \%$ to $98 \% S$. viridis control (Table 7). Halosulfuron alone provided poor $S$. viridis control. Halosulfuron $\left(25,37.5\right.$, and 50 g.ai.ha $\left.{ }^{-1}\right)$ provided a maximum $S$. viridis control of $35 \%$ in white bean (Table 7). $S$-metolachlor (1050 or 1600 g.ai.ha ${ }^{-1}$ ) + halosulfuron $\left(25,37.5\right.$, and $\left.50 \mathrm{~g} \cdot a \mathrm{i} \cdot \mathrm{ha}^{-1}\right)$ controlled $S$. viridis $91 \%$ to $96 \%$ in white bean.

Halosolfuron alone at doses evaluated did not reduce $S$. viridis density or dry weight (Table 7). However, $S$-metolachlor and $S$-metolachlor + halosulfuron reduced $S$. viridis density as much as $89 \%$ and $86 \%$ and $S$. viridis dry weight as much as $94 \%$ and $93 \%$, respectively (Table 7 ). 
Table 7. Percent visible control 4 and 8 WAE, density and dry weight of Setaria viridistreated with $S$-metolachlor and halosulfuron applied PRE at Exeter and Ridgetown $(2016-2018)^{\mathrm{a}, \mathrm{b}}$.

\begin{tabular}{|c|c|c|c|c|c|}
\hline \multirow{2}{*}{ Treatment } & \multirow{2}{*}{$\begin{array}{c}\text { Dose } \\
\left(\mathrm{g} \cdot \mathrm{ai} \cdot \mathrm{ha}^{-1}\right)\end{array}$} & \multicolumn{2}{|c|}{ Control (\%) } & \multirow{2}{*}{$\begin{array}{l}\text { Density } \\
\left(\text { no. } \mathrm{m}^{-2}\right)\end{array}$} & \multirow{2}{*}{$\begin{array}{c}\text { Dry weight } \\
\left(\mathrm{g} \cdot \mathrm{m}^{-2}\right)\end{array}$} \\
\hline & & 4 WAE & $8 \mathrm{WAE}$ & & \\
\hline Weedy control & & $0^{c}$ & $0^{c}$ & $57.7^{\mathrm{c}}$ & $58.1^{\mathrm{c}}$ \\
\hline Weed-free control & & 100 & 100 & $0^{\mathrm{a}}$ & $0^{\mathrm{a}}$ \\
\hline$S$-metolachlor & 1050 & $96^{\mathrm{a}}$ & $96^{\mathrm{a}}$ & $6.6^{\mathrm{b}}$ & $3.8^{\mathrm{b}}$ \\
\hline$S$-metolachlor & 1600 & $98^{\mathrm{a}}$ & $98^{\mathrm{a}}$ & $6.4^{\mathrm{b}}$ & $3.2^{\mathrm{b}}$ \\
\hline Halosulfuron & 25 & $21^{\mathrm{b}}$ & $13^{\mathrm{b}}$ & $47.9^{c}$ & $36.7^{\mathrm{c}}$ \\
\hline Halosulfuron & 37.5 & $25^{\mathrm{b}}$ & $19^{\mathrm{b}}$ & $45.7^{\mathrm{c}}$ & $29.9^{c}$ \\
\hline Halosulfuron & 50 & $35^{\mathrm{b}}$ & $25^{\mathrm{b}}$ & $40.2^{\mathrm{c}}$ & $31.1^{\mathrm{c}}$ \\
\hline$S$-metolachlor + halosulfuron & $1050+25$ & $92^{\mathrm{a}}$ & $91^{\mathrm{a}}$ & $12.4^{\mathrm{b}}$ & $9.1^{\mathrm{b}}$ \\
\hline$S$-metolachlor + halosulfuron & $1050+37.5$ & $92^{\mathrm{a}}$ & $94^{\mathrm{a}}$ & $12.1^{\mathrm{b}}$ & $8.4^{\mathrm{b}}$ \\
\hline$S$-metolachlor + halosulfuron & $1050+50$ & $92^{\mathrm{a}}$ & $92^{\mathrm{a}}$ & $9.6^{\mathrm{b}}$ & $5.8^{\mathrm{b}}$ \\
\hline$S$-metolachlor + halosulfuron & $1600+25$ & $96^{\mathrm{a}}$ & $95^{\mathrm{a}}$ & $8.0^{\mathrm{b}}$ & $5.9^{\mathrm{b}}$ \\
\hline$S$-metolachlor + halosulfuron & $1600+37.5$ & $96^{\mathrm{a}}$ & $95^{\mathrm{a}}$ & $8.5^{\mathrm{b}}$ & $5.2^{\mathrm{b}}$ \\
\hline$S$-metolachlor + halosulfuron & $1600+50$ & $96^{\mathrm{a}}$ & $96^{\mathrm{a}}$ & $8.3 b$ & $3.9 \mathrm{~b}$ \\
\hline
\end{tabular}

${ }^{a}$ Abbreviations: PRE, preemergence; WAE, weeks after white bean emergence. ${ }^{b}$ Means followed by a different letter within a column are significantly different according to a Tukey-Kramer multiple range test at $\mathrm{P}<$ 0.05 .

Other studies have similarly shown $93 \%$ to $97 \% S$. viridis control with $S$-metolachlor [6] [20] and $47 \%$ to $59 \% S$. viridis control with halosufuron in white bean [7] [9]. Li et al. [7] found up to $94 \% S$. viridis control with $S$-metolachlor + halosulfuron at $1050+35 \mathrm{~g} \cdot \mathrm{ai} \cdot \mathrm{ha}^{-1}$.

\section{Conclusions}

There is an adequate margin of crop safety in white bean for use of $S$-metolachlor, halosulfuron and $S$-metolachlor + halosulfuron applied PRE. $S$-metolachlor alone provided poor control of $A$. artemisiifolia, C. album and A. theophrasti, fair control of $A$. retroflexus and excellent control of $S$. viridis and $E$. crus-galli. Halosulfuron alone provided poor control of $C$. album, A. theophras$t i, E$. crus-galli and $S$. viridis, fair control of $A$. artemisiifolia and excellent control of $A$. retroflexus. $S$-metolachlor + halosulfuron tankmixes provided poor control of $C$. album, fair control of $A$. theophrasti, good control of $A$. artemisiifolia and excellent control of $A$. retroflexus, E. crus-galli and $S$. viridis. There was a trend for better control of $A$. artemisiifolia, $C$. album and $A$. theophrasti with the higher doses of halosulfuron. White bean yield with $S$-metolachlor + halosulfuron tankmixes was similar to the weed-free control.

Results also show that the dose of $S$-metolachlor and halosulfuron when applied as a tankmix should be adjusted depending on weeds that exist in the field. 
For fields with $A$. theophrasti, there was a trend for improved control with the higher doses of halosulfuron. For fields with $A$. artemisiifolia, there was a trend for improved control with the higher doses of halosulfuron when applied as a tankmix with the low dose of $S$-metolachlor, however, there was no need to increase the halosulfuron dose when applied as a tankmix with the high dose of $S$-metolachlor. For fields with $A$. retroflexus species, E. crus-galli and $S$. viridis, a tankmix of $S$-metolachlor + halosulfuron at the low dose was sufficient to provide excellent weed control. Using this information, white bean producers can maximize crop yield and reduce input costs while reducing unnecessary loading of herbicides into the environment by adjusting herbicide doses depending on weed species present in their land.

\section{Acknowledgements}

This study was funded in part by Ontario Bean Growers (OBG).

\section{Conflicts of Interest}

The authors declare that there is no conflict of interest regarding the publication of this paper.

\section{References}

[1] Ontario Bean Growers (2019) Ontario Beans. http://ontariobeans.on.ca/types-of-beans

[2] Ontario Ministry of Agriculture, Food and Rural Affairs (2019) Area, Yield, Production and Farm Value of Specified Field Crops (Imperial and Metric Units). http://www.omafra.gov.on.ca/english/stats/crops/index.html

[3] Ontario Ministry of Agriculture, Food and Rural Affairs (2018) Guide to Weed Control. Publication 75. OMAF, Toronto, $355 \mathrm{p}$.

[4] Woolley, B.L., Micheals, T.E., Hall, M.R. and Swanton, C.J. (1993) The Critical Period of Weed Control in White Bean (Phaseolus vulgaris). Weed Science, 41, 180-184. https://doi.org/10.1017/S0043174500076037

[5] Chikoye, D., Weise, S.F. and Swanton, C.J. (1995) Influence of Common Ragweed (Ambrosia artemisiifolia) Time of Emergence and Density on White Bean (Phaseolus vulgaris). Weed Science, 43, 375-380. https://doi.org/10.1017/S0043174500081352

[6] Li, Z., Van Acker, R., Robinson, D.E., Soltani, N. and Sikkema, P.H. (2016) Halosulfuron Tank-Mixes Applied PRE in White Bean (Phaseolus vulgaris L.). Weed Technology, 30, 57-66. https://doi.org/10.1614/WT-D-15-00084.1

[7] Li, Z., Van Acker, R., Robinson, D.E., Soltani, N. and Sikkema, P.H. (2016) Halosulfuron Tank-Mixes Applied Preplant Incorporated for Weed Control in White Bean (Phaseolus vulgaris L.). Canadian Journal of Plant Science, 96, 81-88. https://doi.org/10.1139/cjps-2015-0124

[8] Malik, V.S., Swanton, C.J. and Michaels, T.E. (1993) Interaction of White Bean (Phaseolus vulgaris) Cultivars, Row Spacing, and Seeding Density with Annual Weeds. Weed Science, 41, 62-68. https://doi.org/10.1017/S0043174500057593

[9] Soltani, N., Nurse, R.E., Shropshire, C. and Sikkema, P.H. (2014) Weed Control in 
White Bean with Various Halosulfuron Tankmixes. Advances in Agriculture, 2014, Article ID: 391634. https://doi.org/10.1155/2014/391634

[10] Soltani, N., Nurse, R.E., Shropshire, C. and Sikkema, P.H. (2014) Weed Control with Halosulfuron Applied Preplant Incorporated, Preemergence or Postemergence in White Bean. The Journal of Agricultural Science, 5, 875-881. https://doi.org/10.4236/as.2014.510094

[11] Soltani, N., Dille, J.A., Gulden, R., Sprague, C., Zollinger, R., Morishita, D.W., Lawrence, N.C., Sbatella, G.M., Kniss, A.R., Jha, P. and Sikkema, P.H. (2018) Potential Yield Loss in Dry Bean Crops Due to Weeds in the United States and Canada. Weed Technology, 32, 342-346. https://doi.org/10.1017/wet.2017.116

[12] Wilson, R.G., Wicks, G.A and Fenster, C.R. (1980) Weed Control in Field Beans (Phaseolus vulgaris) in Western Nebraska. Weed Science, 28, 295-299. https://doi.org/10.1017/S0043174500055326

[13] Shaner, D.L. (2014) Herbicide Handbook. 10th Edition, Weed Science Soc. Am., Champaign, $513 \mathrm{p}$.

[14] Silvey, B.D., Mitchem, W.E., Macrae, A.W. and Monks, D.W. (2006) Snap Bean (Phaseolus vulgaris) Tolerance to Halosulfuron PRE, POST, or PRE Followed by POST. Weed Technology, 20, 873-876. https://doi.org/10.1614/WT-05-046.1

[15] O’Connell, P.J., Harms, C.T. and Harms, C.T. (1998) Metolachlor, S-Metolachlor and Their Role within Sustainable Weed-Management. Crop Protection, 17, 207-212. https://doi.org/10.1016/S0261-2194(98)80011-2

[16] Li, Z., Van Acker, R., Robinson, D.E., Soltani, N. and Sikkema, P.H. (2017) Managing Weeds with Herbicides in White Bean in Canada: A Review. Canadian Journal of Plant Science, 97, 755-766. https://doi.org/10.1139/CJPS-2017-0030

[17] SAS Institute Inc. (2016) Base SAS ${ }^{\varpi} 9.4$ Procedures Guide: Statistical Procedures. 5th Edition, SAS Institute Inc., Cary.

[18] Soltani, N., Nurse, R.E., Shropshire, C. and Sikkema, P.H. (2012) Weed Control, Environmental Impact and Profitability of Pre-Plant Incorporated Herbicides in White Bean. American Journal of Plant Sciences, 3, 846-853. https://doi.org/10.4236/ajps.2012.37102

[19] Brown, D. and Masiunas, J. (2002) Evaluation of Herbicides for Pumpkin (Cucurbita spp.). Weed Technology, 16, 282-292. https://doi.org/10.1614/0890-037X(2002)016[0282:EOHFPC]2.0.CO;2

[20] Taziar, A.N., Soltani, N., Shropshire, C., Robinson, D.E, Long, M., Gillard, C.L. and Sikkema, P.H. (2016) Sulfentrazone plus a Low Rate of Halosulfuron for Weed Control in White Bean (Phaseolus vulgaris L.). Agricultural Sciences, 8, 27-238. https://doi.org/10.4236/as.2017.83016 